\title{
Convective Electro-Thermal Simulation of a Generator Circuit Breaker Starting Switch
}

\author{
Marcelo Buffoni' ${ }^{1}$, Francesco Agostini ${ }^{2}$ \\ ${ }^{1}$ ABB Switzerland Ltd. Corporate Research Center \\ Segelhofstrasse 1K, 5405 Baden-Dättwil, Switzerland \\ marcelo.buffoni@ch.abb.com \\ ${ }^{2}$ ABB Switzerland Ltd. High-Voltage Products \\ Elias-Canetti-Strasse 7, 8050 Zürich, Switzerland \\ francesco.agostini@ch.abb.com
}

\section{Extended Abstract}

The increasing technical complexity of power plants and high electricity demands have led to a development of new electrical equipment and special power plant safety features. A key component of a modern power station is the generator circuit breaker (GCB) [1,2], which plays a major role in the power plant protection, offers more operational flexibility and enables the implementation of efficient solutions to reduce the investment cost.

Modern generator switchgear in a gas turbine and combined-cycle power plants also contains switching functions required for the static frequency converter (SFC). The SFC delivers energy during the generator start-up and its output is fed to the generator terminals via the starting switch (SSW) [3]. The SSW consists of six high current fuses enclosed in an aluminium box. Each of the fuses generates significant amounts of heat during its operation and contributes to the temperature rise inside the SSW.

The objective of this work is to simulate the thermal performance during the operation of the starting switch (SSW) under steady state and transient electrical loads. The goal is to verify the thermal limits of the device according to GCB standards and customer requirements. High electrical loads, complex geometry and the compact packaging of the SSW require a three-dimensional, transient, coupled electromagnetic-conjugate heat transfer (CHT) simulation, in order to understand the exact thermal performance of the device.

In the case at hand, the main flow driver is the buoyancy force induced by the density gradients in the air, which surrounds the loss-generating electrical parts. The main thermal phenomena taken into account are the natural convection, heat conduction and heat radiation. The internal geometry of the previously mentioned high current fuses is complex and, thus, unfeasible to resolve by meshing. Hence, a thermal model of the fuse based on a thermal resistors methodology is computed and used in during the CHT calculation. The CHT simulations are validated with experiments and a close correlation between them is found. The fuse model is verified against data sheets provided by the supplier.

The CHT results reveal the thermal behavior of the SSW under steady and transient load cycles. Moreover, analysis of the velocity and temperature fields is presented. Thus, a new design of the SSW is proposed in order to obtain better thermal operation of the device, as well as to reach a lower cost of manufacturing.

\section{References}

[1] ABB Switzerland Ltd. [Online] Available: https://new.abb.com/high-voltage/gcb/modules/hec-10-up-to-210-ka

[2] ABB Switzerland Ltd. [Online] Available: https://library.e.abb.com/public/6a55032d57bd43828623ff83ee61ca50/Generator\%20circuitbreaker\%20portfolio\%20brochure_2015.pdf

[3] ABB Switzerland Ltd. [Online] Available: https://library.e.abb.com/public/230e0cabcd374480a4521dbda47c4efb/HVS\%20brochure.pdf 